\title{
Alcohol Use Disorder is Associated with an Increased Risk for Respiratory Failureamong Patients with Takotsubo Cardiomyopathy
}

\author{
Temidayo Abe ${ }^{1 *}$, Valery Effoe ${ }^{2}$, Dolphurs Hayes ${ }^{3}$, Obiora Egbuche $^{2}$, Huynh Ky $^{3}$, Taiwo Ajose ${ }^{1}$, Adebanjo Olowu $^{4}$ \\ ${ }^{1}$ Internal Medicine Residency Program, Morehouse School of Medicine, Atlanta, Georgia 30310, United States; ${ }^{2}$ Department \\ of Cardiovascular Disease, Morehouse School of Medicine, Atlanta, Georgia 30310, United States; ${ }^{3}$ Department of Medicine, \\ Morehouse School of Medicine, Atlanta, Georgia 30310, United States; ${ }^{4}$ JHPIEGO, Plot 971 Reuben Okoye Crescent, Wuye, \\ Abuja, Nigeria
}

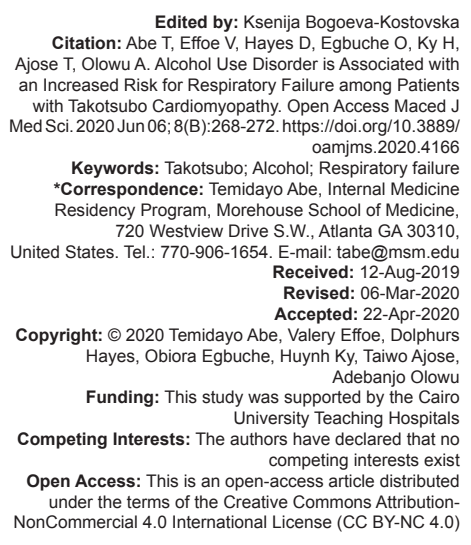

Introduction

Takotsubo cardiomyopathy (TCM) or stressinduced cardiomyopathy is a transient reversible myocardial dysfunction thought to be secondary to exaggerated myocardial catecholamine exposure [1]. Predominantly seen in post-menopausal female in the setting of emotional or physical stressors, it is an increasingly recognized cause of inpatient hospitalization in recent years [1], [2]

Although, initially considered a benign condition, multiple observational studies have consistently demonstrated increased mortality and morbidity in patients with TCM [3]. Notably, up to $20 \%$ of these patients will go on to develop cardiogenic shock, up to $15 \%$ will develop a cardiac arrhythmia, and $4.5 \%$ will develop stroke. Finally, the in-hospital mortality rate can be as high as 5\% [3], [4]. In relation, it is important to determine the predictors of poor outcomes in this patient population, to allow appropriate risk stratification, mitigate the risks, and improve overall outcomes.
Clinical factors such as older age, physical stressors, diabetes mellitus, and pre-existing heart failure have been associated with poor outcomes [4]. The impact of alcohol use on outcomes in patients with TCM has not been discussed. Oxidative stress and accelerated protein catabolism have been implicated in the pathogenesis of alcoholic cardiomyopathy [5], [6]. In addition, they might exacerbate pre-existing heart conditions which may delay recovery and lead poor outcomes in patients with TCM [5], [6]. We aim to determine the effects of alcohol use on outcomes among patients with TCM.

\section{Methods}

\section{Study design}

This is a cross-sectional study that used the discharge data from the National Inpatient Sample (NIS) 
database for the year 2009-2012. The NIS, Healthcare Cost and Utilization Project, agency for healthcare research and quality (AHRQ) is the largest all-payer inpatient database in the United States (US). The database contains a $20 \%$ stratified sample of all discharges from the US nonfederal short-term general hospitals, subspecialty hospitals, and public hospitals and is stratified based on the number of beds, ownership, hospital teaching status, US region, and state. Stratified random sampling ensures that the database is representative of the US population and accounts for $90 \%$ of all hospitalizations in the US after applying appropriate weights. The NIS includes information on demographic characteristics, hospital characteristics, up to 25 diagnostic and procedure codes based on the International Classification of Diseases $9^{\text {th }}$ revision, Clinical Modification (ICD-9-CM), and outcomes based on patient discharge records. Each record represents a single hospitalization and thus, multiple records are possible for an individual with recurrent hospitalizations [7].

\section{Study population, variables, and outcomes}

We identified patients aged 18-75 years from the 2009 to 2012 NIS with the discharge diagnosis of TCM using ICD-9-CM code 429.83 (Figure 1). The study group consisted of patients with a diagnosis of both TCM and alcohol use disorder (AUD), while the control group included - patients with a diagnosis of TCM without AUD in any of their discharge codes. AUD was identified using the ICD-9-CM codes 305.00-305.03 and 303.90-303.93 (supplementary Table 1). Demographics and primary prayer associated with each discharge diagnosis were identified from the NIS. Comorbidities were identified using the $A H R Q$ comorbidity software. This software allows identification of comorbidities not related to the primary diagnosis that was present before admission [8]. Comorbidities chosen were those used in prior studies evaluating outcomes in patients with TCM [8], [9], [10]. The primary outcomes of interest included overall in-hospital mortality, atrial fibrillation, sudden cardiac arrest, cardiogenic shock, acute respiratory failure, stroke, and mechanical hemodynamic support (MHS). All clinical variables were defined by the ICD9-CM codes (See supporting information for ICD-9-CM codes of these variables).

\section{National Inpatient Sample 2009 - 2012}

Indentified 11,221 patients with a discharge diagnosis of TCM using ICD-9-CM code 429.83

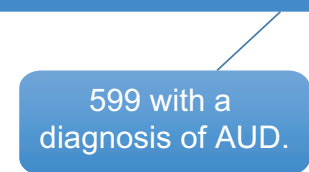

10,622 without a diagnosis of AUD.

\section{Statistical analysis}

All statistical analyses were performed using SPSS version 20. We compared baseline patient characteristics and outcomes between patients with concomitant TCM and AUD and those with TCM without AUD. Chi-square test of significance was used for categorical variables and independent sample t-test to compare for the continuous variable. Logistic regression analysis was used to estimate the adjusted odds ratios (aOR) the outcomes between these two groups patients. Covariates adjusted for were include age, sex, tobacco use, hypothyroidism, chronic kidney disease, obesity, chronic obstructive pulmonary disease, diabetes mellitus, hyperlipidemia, and coronary artery disease. Association of each outcome variable with AUD was reported as aOR with 95\% confidence interval. A twotailed $p<0.05$ was considered statistically significant.

\section{Results}

\section{Baseline demographics}

We identified 11,221 patients with a discharge diagnosis of TCM. Among these patients, 599 (5.3\%) had AUD and 10,622 (94.6\%) did not have AUD. The study sample was mostly female $(86.6 \%$, Table 1$)$. The mean age of patients with AUD was significantly lower compared to patient without AUD (56.01 \pm 11 vs. $60.47 \pm 11$; $p<0.0001)$. There were no significant racial differences between both groups. The prevalence of tobacco use disorder $(45.9 \%$ vs. $19 \%$; $p<0.0001)$ was significantly

Table 1: Baseline characteristics of study participants by alcohol use disorder status

\begin{tabular}{|c|c|c|c|}
\hline Demographics & TCM ALONE $(10,622)$ & TCM + AUD (599) & $\mathrm{p}$-value \\
\hline Age (years), mean (SD) & $60.47(11)$ & $56.01(11)$ & $<0.0001$ \\
\hline Sex & & & $<0.0001$ \\
\hline Female (n, \%) & $9297(87.4)$ & $427(71.3)$ & \\
\hline Male (n, \%) & 1335 (12.6) & $172(28.7)$ & \\
\hline Race & & & 0.159 \\
\hline White (n, \%) & $7629(81.0)$ & $447(83.4)$ & \\
\hline Black (n, \%) & $725(7.7)$ & $45(8.4)$ & \\
\hline Hispanic $(\mathrm{n}, \%)$ & $552(5.9)$ & $20(3.7)$ & \\
\hline Asian or Pacific Islander ( $\mathrm{n}, \%)$ & $187(2.0)$ & $5(0.9)$ & \\
\hline Native America (n, \%) & $68(0.7)$ & $5(0.9)$ & \\
\hline Other (n, \%) & $261(2.8)$ & $14(2.6)$ & \\
\hline Primary prayer & & & $<0.0001$ \\
\hline Medicare (n, \%) & $5093(48.0)$ & $207(34.7)$ & \\
\hline Medicaid (n, \%) & $999(9.4)$ & $110(18.4)$ & \\
\hline Private insurance $(\mathrm{n}, \%)$ & $3649(34.4)$ & $169(28.3)$ & \\
\hline Self-pay (n, \%) & $522(4.9)$ & $77(12.9)$ & \\
\hline No charge $(n, \%)$ & $45(0.4)$ & $4(0.7)$ & \\
\hline Other $(n, \%)$ & $308(2.9)$ & $30(5.0)$ & \\
\hline \multicolumn{4}{|l|}{ Comorbidities } \\
\hline Tobacco use disorder (n, \%) & $2023(19)$ & $275(45.9)$ & $<0.0001$ \\
\hline $\operatorname{VHD}(n, \%)$ & $82(0.8)$ & $3(0.5)$ & 0.457 \\
\hline Hypothyroidism (n, \%) & $1527(14.4)$ & $62(10.4)$ & 0.006 \\
\hline CKD $(n, \%)$ & $850(8.0)$ & $20(3.3)$ & $<0.0001$ \\
\hline Obesity (n, \%) & $1136(10.7)$ & $28(4.7)$ & $<0.0001$ \\
\hline $\mathrm{CHF}(\mathrm{n}, \%)$ & $2856(26.9)$ & $172(28.7)$ & 0.32 \\
\hline $\operatorname{COPD}(\mathrm{n}, \%)$ & $2116(19.9)$ & $140(23.4)$ & 0.039 \\
\hline Hyperlipidemia (n, \%) & $3882(36.5)$ & $117(19.5)$ & $<0.0001$ \\
\hline Diabetes $(n, \%)$ & $2640(24.8)$ & $112(18.7)$ & 0.001 \\
\hline Hypertension (n, \%) & $6380(60.0)$ & $339(56.6)$ & 0.097 \\
\hline $\operatorname{CAD}(\mathrm{n}, \%)$ & $3660(34.4)$ & $182(30.4)$ & 0.043 \\
\hline
\end{tabular}

Figure 1: Selection of the patients 
higher in patients with AUD, while the prevalence of chronic kidney disease $(3.3 \%$ vs. $8 \%$; $p<0.0001)$, obesity (4.7\% vs. 10.7\%; p < 0.0001), hyperlipidemia (19.5\% vs. $36.5 \%$; $p<0.0001)$, diabetes mellitus $(18.7 \%$ vs. $24.8 \% ; p=0.001)$, and hypothyroidism (10.4\% vs. $14.4 \% ; p=0.006$ ) were significantly lower (Table 1$)$.

\section{Outcomes}

There was no significant difference between the two groups in the rates of atrial fibrillation $(10.4 \%$ vs. $8,5 \%$; $p=0.134)$, cardiogenic shock (5.9\% vs. $4.8 \%$; $p=0.3)$, use of MHS (2.6\% vs. $1.7 \% ; p=0.165)$, overall in

Table 2: Outcomes in patients with alcohol use disorder compared to those without alcohol use disorder

\begin{tabular}{llll}
\hline Outcomes & TCM alone & TCM+AUD & -value \\
\hline Overall mortality (n, \%) & $425(4.0)$ & $22(3.7)$ & 0.691 \\
Mechanical hemodynamic support (n, \%) & $275(2.6)$ & $10(1.7)$ & 0.165 \\
Atrial fibrillation $(\mathrm{n}, \%)$ & $1109(10.4)$ & $51(8.5)$ & 0.134 \\
Respiratory failure $(\mathrm{n}, \%)$ & $1880(17.7)$ & $151(25.2)$ & $<0.0001$ \\
Cardiac arrest $(\mathrm{n}, \%)$ & $287(2.7)$ & $21(3.5)$ & 0.240 \\
Cardiogenic shock (n, \%) & $623(5.9)$ & $29(4.8)$ & 0.300 \\
Stroke $(\mathrm{n}, \%)$ & $172(1.6)$ & $8(1.3)$ & 0.593 \\
\hline TCM: Takotsubo cardiomyopathy; AUD: Alcohol use disorder. $\mathrm{p}<0.05$ means statistical significant. Rates \\
of sudden cardiac arrest and acute respiratory failure was significantly higher in patients with alcohol use
\end{tabular}
of sudden

hospital mortality (4.0\% vs. $3.7 \% ; p=0.691)$, stroke (1.6\% vs. $1.3 \% ; p=0.593)$, and sudden cardiac death (2.7\% vs. $3.5 \% ; p=0.24)$. Rates of acute respiratory failure $(25.2 \%$, vs. $17.7 \% ; p<0.0001)$ were significantly higher in patients with AUD compared to those without AUD (Table 2 and Figure 2). After adjusting for covariates (Table 3 ), the

Table 3: Adjusted odds ratios and $95 \%$ confidence intervals for outcomes among those with alcohol use disorder compared to those without

\begin{tabular}{lllll}
\hline Outcomes & TCM & aOR $(95 \%$ Cl $)$ & TCM+AUD & p-value \\
\hline Overall mortality & 1.0 & & $0.81(0.52-1.26)$ & 0.34 \\
AF & 1.0 & & $1.21(0.89-1.65)$ & 0.22 \\
Cardiac arrest & 1.0 & & $1.13(0.71-1.78)$ & 0.61 \\
Cardiogenic shock & 1.0 & & $0.73(0.50-1.08)$ & 0.13 \\
MHS & 1.0 & $0.59(0.31-1.11)$ & 0.10 \\
Respiratory failure & 1.0 & & $1.34(1.11-1.66)$ & 0.003 \\
Stroke & 1.0 & $0.72(0.35-1.48)$ & 0.36 \\
\hline AUD: Alcohol use disorder: Cl: Confidence interval; AF: Atrial fibrillation: MHS: Mechanical hemodynamic
\end{tabular}

AUD: Alcohol use disorder; Cl: Confidence interval; AF: Atrial fibrillation; MHS: Mechanical hemodynamic support; Adjusted covariates include age, sex, tobacco use, hypothyroidism, chronic kidney disease, obesity, chronic obstructive pulmonary disease, diabetes mellitus, hyperlipidemia, and coronary artery disease.

odds ratio for acute respiratory failure (aOR 1.36; $95 \% \mathrm{Cl}$ : 1.11-1.66) in patients with AUD compared to those without AUD remained significant. The association for the other outcomes was attenuated when adjusted for covariates.

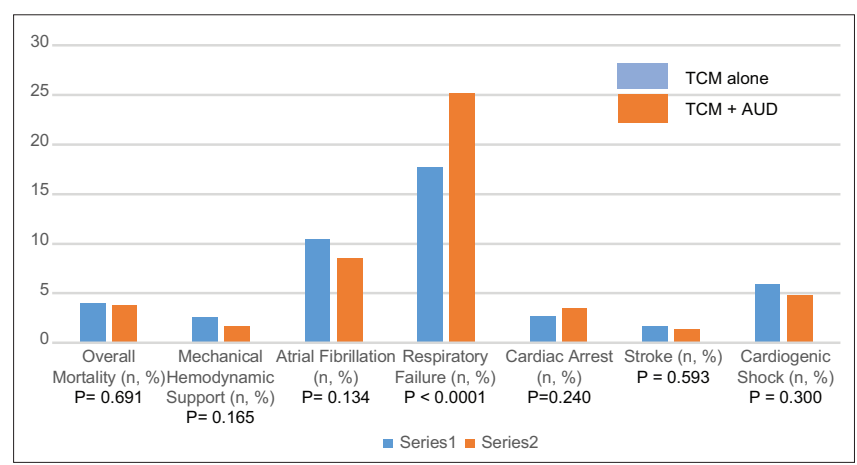

Figure 2: Proportion of outcomes by alcohol use disorder status. The vertical axis represents percentage of outcomes, while the horizontal axis, the specified outcomes, and corresponding p-values. TCM: Takotsubo cardiomyopathy, AUD: Alcohol use disorder

\section{Discussion}

In this study, we investigated the association between concomitant TCM and AUD compared to TCM without AUD and outcomes. We found a significant association between concomitant TCM and AUD and acute respiratory, with an estimated $36 \%$ increased risk, compared to those with TCM without AUD. There was no apparent association between concomitant TCM and AUD, and atrial fibrillation, stroke, sudden cardiac death, use of MHS, cardiogenic shock, or overall inpatient mortality. To our knowledge, this is the first study to evaluate the effects of AUD on TCM outcomes.

We have seen an increased incidence in TCM in recent years due to increased awareness and the wide availability of invasive coronary angiography [1]. While it was previously considered benign, recent studies have demonstrated rates of in-hospital complications similar to those in patients with acute coronary syndrome [11]. Ghadri et al. reported up to $15 \%$ rate of atrial fibrillation, $20 \%$ cardiogenic shock, and $6 \%$ sudden cardiac arrest in these patients [4]. Common predictors of these outcomes include a physical stressor, age $>75$ years, male sex, reduction in left ventricular systolic function on admission, high brain natriuretic peptide levels, and a troponin $>10$ times the upper reference limit [4]. In a recent study, Desai et al. demonstrated an increased risk of cardiac complications in obese patients with TCM compared to non-obese patients [12].

Acute respiratory failure as an in-hospital complication in TCM has not been well studied. Prior studies looking at in-hospital complications in patients with TCM have focused on cardiac complications such as cardiogenic shock, cardiac arrest, myocardial infarction, and arrhythmias [11]. In our study, we found an $18 \%$ rate of acute respiratory failure among patients with TCM, which is higher than that reported in other studies evaluating acute respiratory failure as a complication of TCM [8], [9], [12]. Desai et al., while evaluating TCM complications in obese compared to non-obese patients, observed a $12 \%$ rate of acute respiratory failure, while Yassin et al. observed a 14\% rate in another study evaluating the impact of atrial fibrillation on TCM [8], [12]. In our study, we found a $25 \%$ prevalence of acute respiratory failure in patients with concomitant TCM and AUD, compared to $17.7 \%$ in those without AUD.

While the exact pathogenesis of TCM is unclear, it is thought to be secondary to exaggerated myocardial catecholamine exposure [3]. Furthermore, studies have suggested that oxidative stress may also play a role through inducing left ventricular dysfunction by affecting calcium homeostasis and myocardial contractile function [10], [13]. Chronic alcohol use weakens the pulmonary immune system and makes the cells more susceptible to oxidative stress injury, increasing the risk for acute respiratory distress syndrome and 
pneumonia[14],[15],[16]. Another plausible explanation for the higher risk of acute respiratory failure seen in patients with concomitant TCM and AUD is that patients with AUD have an increased risk of aspiration pneumonia, which can eventually lead to acute respiratory failure [17]. Furthermore, oxidant injury might explain acute respiratory failure seen in patients with TCM and increased susceptibility to such injury might explain why the rates are higher in patients with AUD.

\section{Limitations}

Our study has some limitations related to the NIS database: (1) We identified patients with AUD using ICD-9-CM codes. We might have missed some patients with this condition in cases where the diagnosis was not coded. Furthermore, we were unable to quantify alcohol intake. (2) Information on laboratory and clinical data such as vital signs and left ventricular ejection fraction to determine the severity of TCM, was unavailable. This might play a role on the outcomes.

\section{Conclusion}

Our study showed that the risk of acute respiratory failure was significantly higher among patients with concomitant TCM and AUD compared to those without AUD. Future studies are needed to replicate our findings and further clarify the impact of AUD on outcomes in patients with TCM.

\section{References}

1. Y-Hassan S, Tornvall P. Epidemiology, pathogenesis, and management of takotsubo syndrome. Clin Auton Res. 2018;28(1):53-65. https://doi.org/10.1007/s10286-017-0465-z PMid:28917022

2. Lyon AR, Bossone E, Schneider B, Sechtem U, Citro R, Underwood SR, et al. Current state of knowledge on takotsubo syndrome: A position statement from the taskforce on takotsubo syndrome of the heart failure association of the European society of cardiology. Eur J Heart Fail. 2015;18(1):8-27. https:// doi.org/10.1002/ejhf.424

PMid:26548803

3. Ghadri JR, Wittstein IS, Prasad A, Sharkey S, Dote K, Akashi YJ, et al. International expert consensus document on takotsubo syndrome (Part I): Diagnostic workup, outcome, and management. Eur Heart J. 2018;39(22):2032-46. https://doi. org/10.1093/eurheartj/ehy077

PMid:29850871

4. Ghadri JR, Wittstein IS, Prasad A, Sharkey S, Dote K, Akashi YJ, et al. International expert consensus document on takotsubo syndrome (Part II): Diagnostic workup, outcome, and management. Eur Heart J. 2018;39(22):2047-62. https://doi. org/10.1093/eurheartj/ehy077

PMid:29850820

5. Steiner JL, Lang $\mathrm{CH}$. Etiology of alcoholic cardiomyopathy: Mitochondria, oxidative stress and apoptosis. Int J Biochem Cell Biol. 2017;89:125-35. https://doi.org/10.1016/j. biocel.2017.06.009

PMid:28606389

6. Piano MR, Phillips SA. Alcoholic cardiomyopathy: Pathophysiologic insights. Cardiovasc Toxicol. 2014;14(4):291308. https://doi.org/10.1007/s12012-014-9252-4 PMid:24671642

7. Anand V, Roy SS, Archer SL, Weir EK, Garg SK, Duval S, et al. Trends and outcomes of pulmonary arterial hypertensionrelated hospitalizations in the United States: Analysis of the nationwide inpatient sample database from 2001 through 2012. JAMA Cardiol. 2016;1(9):1021-29. https://doi.org/10.1001/ jamacardio.2016.3591

PMid:27851838

8. Yassin AS, Subahi A, Adegbala O, Abubakar H, Dawdy J, Mishra T, et al. Clinical impact of atrial fibrillation on short-term outcomes and in-hospital mortality in patients with takotsubo syndrome: A propensity-matched national study. Cardiovasc Revasc Med. 2019;2019:S1553-8389. https://doi.org/10.1016/j. carrev.2019.07.022

PMid:31439442

9. Lemor A, Ramos-Rodriguez AJ, De La Villa R, Dehkordi SH, Lara FV, Lee S, et al. Impact of gender on in-hospital outcomes in patients with takotsubo syndrome: A nationwide analysis from 2006 to 2014. Clin Cardiol. 2019;42(1):13-8. https://doi. org/10.1002/clc.23109

PMid:30393886

10. Gupta S, Goyal P, Idrees S, Aggarwal S, Bajaj D, Mattana J. Association of endocrine conditions with takotsubo cardiomyopathy: A comprehensive review. J Am Heart Assoc. 2018;7(19):e009003. https://doi.org/10.1161/jaha.118.009003 PMid:30371307

11. Pelliccia F, Pasceri V, Patti G, Tanzilli G, Speciale G, Gaudio C, et al. Long-term prognosis and outcome predictors in takotsubo syndrome. A systematic review and meta-regression study. JACC Heart Fail. 2019;7(2):143-54. https://doi.org/10.1016/j. jchf.2018.10.009 PMid:30611720

12. Desai R, Singh S, Baikpour M, Goyal H, Dhoble A, Deshmukh A, et al. Does obesity affect the outcomes in takotsubo cardiomyopathy? Analysis of the nationwide inpatient sample database, 2010-2014. Clin Cardiol. 2018;41(8):1028-34. https:// doi.org/10.1002/clc.22999 PMid:29917260

13. Roshanzamir S, Showkathali R. Takotsubo cardiomyopathy a short review. Curr Cardiol Rev. 2013;9(3):191-6. https://doi. org/10.2174/1573403×11309030003 PMid:23642025

14. Molina PE, Gardner JD, Souza-Smith FM, Whitaker AM. Alcoho abuse: Critical pathophysiological processes and contribution to disease burden. Physiology (Bethesda). 2014;29(3):203-15. https://doi.org/10.1152/physiol.00055.2013 PMid:24789985

15. Moss M, Bucher B, Moore FA, Moore EE, Parsons PE. The role of chronic alcohol abuse in the development of acute respiratory distress syndrome in adults. JAMA. 1996;275(1):50-4. https:// doi.org/10.1001/jama.1996.03530250054027 PMid:8531287

16. Gupta NM, Lindenauer PK, Yu PC, Imrey PB, Haessler S, Deshpande A, et al. Association between alcohol use disorders and outcomes of patients hospitalized with community-acquired 
pneumonia. JAMA Netw Open. 2019;2(6):e195172. https://doi. org/10.1001/jamanetworkopen.2019.5172

PMid:31173120

17. Kornum JB, Due KM, Nørgaard M, Tjønneland A, Overvad K,
Sørensen HT, et al. Alcohol drinking and risk of subsequent hospitalisation with pneumonia. Eur Respir J. 2012;39(1):14955. https://doi.org/10.1183/09031936.00000611

PMid:21659417

\section{Supplementary Table 1}

International Classification of Disease 9th Revision -Clinical Modification Codes (ICD-9 CM) and Clinical Classifications Software and procedure (PR) codes used for Comorbidities and Complications not provided by NIS database

(The comorbidity codes provided by database: https://www.hcup-us.ahrq.gov/toolssoftware/comorbidity/ Table2-FY2010-V3_5.pdf)

\begin{tabular}{ll}
\hline Comorbidities & ICD-9 CM/CCS/PR codes \\
Tobacco use disorder & 305.1 \\
Valvular heart disease & $424.0,424.1,424.2,424.3$ \\
Hypothyroidism & 244.9 \\
Chronic kidney disease & $585.1-585.5$ \\
Obesity & $278.0-278.03$ \\
Hypertension & 401.9 \\
Congestive heart failure & 428.0 \\
Chronic obstructive pulmonary disease & 491,492 \\
Diabetes & $250.00-250.03$ \\
Hyperlipidemia & 272.4 \\
Coronary artery disease & 414.01 \\
Complications & \\
Stroke & 434.91 \\
Atrial fibrillation & 427.31 \\
Cardiogenic shock & 785.51 \\
Cardiac arrest & 427.5 \\
Mechanical circulatory support (percutaneous ventricular & PR 37.68, PR 37.61 \\
assist device (Impella@), intra-aortic balloon pump & \\
Respiratory failure & 518.81 \\
\hline
\end{tabular}

